\title{
Altered capicua transcriptional repressor gene expression exhibits distinct prognostic value for isocitrate dehydrogenase-mutant oligodendroglial tumors
}

\author{
FUXIN HAN $^{1 *}$, JINSONG ZHANG $^{2 *}$, SHANBO MA $^{3,4 *}$, XIAOYAN CHEN $^{1}$, \\ WEIPING LIU ${ }^{1}$, XIAOSHENG HE ${ }^{1}$, ZHOU FEI $^{1}$ and YANGANG WANG ${ }^{1}$
}

Departments of ${ }^{1}$ Neurosurgery, ${ }^{2}$ Radiology and ${ }^{3}$ Pharmacy, Xijing Hospital, Fourth Military Medical University, Xi'an, Shaanxi 710032; ${ }^{4}$ School of Pharmacy, Shaanxi University of Chinese Medicine, Xianyang, Shaanxi 712000, P.R. China

Received June 13,2016; Accepted August 15, 2017

DOI: $10.3892 / \mathrm{ol} .2017 .7460$

\begin{abstract}
To evaluate the prognostic significance of the altered expression of capicua transcriptional repressor (CIC) in isocitrate dehydrogenase $(I D H)$-mutant oligodendroglial tumors, a cohort of $54 \mathrm{IDH}$-mutant oligodendroglial tumors (designated as the Xijing cohort) were examined by immunohistochemistry (IHC), and two public expression data sets from The Cancer Genome Atlas (TCGA; $n=265$ ) and the Gene Expression Omnibus (GEO; $\mathrm{n}=45$ ) were analyzed in the present study. The prognostic value was evaluated by survival analysis and Cox hazards models. Overall survival (OS) was investigated with Kaplan-Meier curves and log-rank tests. Gene set enrichment analysis (GSEA) was also performed to characterize the functional profiles of each subgroup. It was revealed that in $I D H$-mutant, $1 \mathrm{p} / 19 \mathrm{q}$ co-deleted oligodendroglial tumors, higher CIC expression (at mRNA and protein levels) was associated with a more favorable OS time (log-rank P-values: TCGA, $\mathrm{P}=0.034$; $\mathrm{GEO}, \mathrm{P}=0.012$; Xijing cohort, $\mathrm{P}=0.029)$. By contrast, among $I D H$-mutant, $1 \mathrm{p} / 19 \mathrm{q}$ intact tumors, higher CIC expression was associated with poorer OS time (log-rank P-values: TCGA, $\mathrm{P}=0.007$; GEO, $\mathrm{P}=0.017$; Xijing cohort, $\mathrm{P}=0.012$ ). To the best of our knowledge, this is the first study demonstrating the distinct prognostic value of altered CIC expression with regard to the $1 \mathrm{p} / 19 \mathrm{q}$ status among $I D H$-mutant oligodendroglial tumors. The dual roles of CIC may be influenced by its transcriptional regulatory activity
\end{abstract}

Correspondence to: Professor Yangang Wang or Professor Zhou Fei, Department of Neurosurgery, Xijing Hospital, Fourth Military Medical University, 127 Changle West Road, Xi'an, Shaanxi 710032, P.R. China

E-mail: ygwangfmmu@126.com

E-mail: feizhou@fmmu.edu.cn

*Contributed equally

Key words: oligodendroglial tumors, isocitrate dehydrogenase mutations, 1p/19q co-deletion, capicua transcriptional repressor gene, prognostic value and the consequent functional profiles. Additionally, a simple risk classification scheme based on CIC expression alone is proposed for the optimal prediction of prognosis in patients with oligodendroglial tumors.

\section{Introduction}

Oligodendroglial tumors, including oligodendrogliomas (ODs) and oligoastrocytomas (OAs), present as distinct histopathological and clinical subsets of glioma, with slow growth and a favorable prognosis $(1,2)$. Oligodendroglial tumors account for $<10 \%$ of the diffuse gliomas (2). The histopathological diagnosis of these tumors is difficult: OAs with typical morphological features are histologically well defined, whereas other non-classical tumors, OAs in particular, are more challenging to diagnose due to equivocal definitions as a result of sharing oligodendroglial and astrocytic morphology, as well as the uncertainty of representative tumor sampling $(2,3)$. Therefore, histological diagnosis provides inadequate prognostic information on oligodendroglial tumors, as demonstrated by markedly varied survival times among patients with similar histological features in the clinic (2).

Like other tumors, oligodendroglial tumors develop as a result of heterogeneous genetic and molecular alterations (1). These tumor-specific molecular abnormalities determine the clinical and biological behavior of each tumor; therefore certain molecular biomarkers may provide useful clinical information (2). At present, two identified biomarkers for oligodendroglial tumors are mutations in isocitrate dehydrogenase $(I D H) 1$ and $I D H 2$ (referred to collectively as $I D H$ ), and the loss of heterozygosity of chromosome arms $1 \mathrm{p}$ and $19 \mathrm{q}(2)$. $I D H$ mutations are observed in $70-80 \%$ of oligodendroglial tumors and are associated with an improved survival time (2). The complete co-deletion of $1 \mathrm{p} / 19 \mathrm{q}$ resulting from an unbalanced translocation is present in $60-80 \%$ of ODs, whereas it is observed less frequency in OAs (10-15\%) (4); this genetic alteration is associated with a positive outcome and response to chemotherapy (5). The majority of $1 \mathrm{p} / 19 \mathrm{q}$ co-deleted oligodendroglial tumors also exhibit IDH mutations (6).

Sequencing studies have identified recurrent mutations in the Capicua transcriptional repressor $(C I C)$ gene 
within $50-70 \%$ (2) of $1 \mathrm{p} / 19 \mathrm{q}$ co-deleted oligodendroglial tumors (1,3,4,7-10). CIC is situated on cytoband $19 \mathrm{q} 13.2$ and encodes a $164 \mathrm{kDa}$ protein containing a high-mobility group box (9). The gene was first identified as a downstream repressor of the receptor tyrosine kinase signaling pathway in regulating developmental patterning and cell fate in Drosophila (11). Mutations in this gene are almost exclusively observed in oligodendroglial tumors (3,7). Certain mutations may result in CIC repression; tumors with this mutation comprise an aggressive subset of 1q/19q co-deleted gliomas (4). However, there are inconsistent reports regarding the association between CIC mutations and prognosis in patients with oligodendroglial tumors; some reported a significant survival correlation of CIC mutations whiles others did not $(4,8,9)$. Therefore, the aim of the present study was to investigate the prognostic ability of altered CIC expression level as an alternative to CIC mutation status among $I D H$-mutant oligodendroglial tumors with or without co-deletion of $1 \mathrm{p} / 19 \mathrm{q}$.

\section{Patients and methods}

Patients and samples. Data and samples from patients with glioma who were admitted between January 2003 and December 2012 to the Department of Neurosurgery, Xijing hospital (Xi'an, China) were reviewed in the present study. A total of 54 patients who met the following inclusion criteria were included in the present study: i) Aged >18 years; ii) with pathologically confirmed oligodendroglial tumors, including ODs and OAs [World Health Organization (WHO) grade II to III]; iii) carrying $I D H 1$ mutations (as determined by anti-IDH1 ${ }^{\mathrm{R} 132 \mathrm{H}}$ immunohistochemistry (IHC) analysis at the Department of Pathology, Xijing hospital); iv) with available records of $1 \mathrm{p} / 19 \mathrm{q}$ status (determined by fluorescence in situ hybridization analysis at the Department of Pathology, Xijing hospital); and v) with written informed consent. The histopathologic features and WHO grading were evaluated according to the WHO 2007 classification (12). Formalin-fixed, paraffin-embedded tumor tissues were examined in the present study. All patients underwent the radical neurosurgery of primary tumors and received stereotactic fractionated radiosurgery, with a median total dose of $36 \mathrm{~Gy}$, postoperatively. Patient demographics and clinical data were retrieved from the Xijing Hospital electronic medical record system. Local institutional review board approval was obtained to use archived material for study purposes. All procedures performed with human tissue were in accordance with the ethical standards of the institutional research committee and with the 1964 Helsinki declaration and its later amendments, or comparable ethical standards.

IHC staining for CIC protein. The protein expression of CIC was examined by IHC staining. In the literature, the vast majority of mutations in CIC occurred in exons 5 and $20(3,9)$. Therefore, an antibody specific for the C-terminus region of CIC protein (cat. no. TA590520; OriGene Technologies, Inc., Rockville, MD, USA) was used. IHC staining was performed as described previously (13); briefly, paraffin-embedded sections were deparaffinized and rehydrated, and antigen retrieval was performed. Subsequently the tumor sections were immersed in methanol containing $3.0 \%$ hydrogen peroxide for $20 \mathrm{~min}$ to block endogenous peroxidase activity, and incubated in $2.5 \%$ goat serum (Abcam, Cambridge, UK) to decrease non-specific binding. Thereafter, tumor sections were incubated at $4^{\circ} \mathrm{C}$ overnight with the primary anti-human CIC antibody (dilution, 1:1,000) followed by incubation with a horseradish peroxidase-conjugated goat anti-rabbit IgG antibody (dilution, 1:1,200; cat. no. A32732; Pierce; Thermo Fisher Scientific, Inc., Waltham, MA, USA) for $30 \mathrm{~min}$. Immunolabeled sections were visualized by staining with 3,3'-diaminobenzidine (Sigma-Aldrich; Merck KGaA, Darmstadt, Germany) and counterstaining with hematoxylin. Negative controls were sections acquired from the same tumor without primary antibody incubation.

The CIC nuclear staining pattern was evaluated in a semiquantitative manner, as previously described (9). Briefly, the protein levels were scored by multiplying the degree of intensity by percentage of stained cells, with the intensity being graded as 0 (no staining), 1 (weak), 2 (moderate) and 3 (strong), and the percentage of stained cells being classified as $0(<10 \%), 1(10-50 \%)$ and $2(>50 \%)$. The CIC scoring was performed by two independent investigators, with any scoring disputes resolved through discussion. Investigators were blinded to the clinical data of the assessed sections, but not to the objectives of the study. The scores were designated as absent (0), weak (1 and 2), moderate (3 and 4) or strong (6).

\section{Public datasets of oligodendroglial tumors}

The cancer genome atlas (TCGA). Clinical and demographical data from 265 patients ( $\geq 18$ years old) with oligodendroglial tumors carrying $I D H$ mutations were retrieved from the TCGA data portal (https://tcga-data.nci.nih.gov/). Somatic mutations in $I D H$ and $C I C$ were determined by level 2 Illumina Genome Analyzer DNA Sequencing data. For the assessment of the 1p/19q status, level 3 Affymetrix Genome-Wide Human SNP 6.0 array data were analyzed within the GISTIC 2.0 module at GenePattern (http://genepattern.broadinstitute.org/gp). An amplitude threshold of \pm 0.2 was used. Additionally, CIC mRNA expression levels were determined using level 3 IlluminaHiSeq_RNASeqV2 data.

Gene expression omnibus (GEO). Data from a total of 45 patients ( $\geq 18$ years old) with oligodendroglial tumors carrying an IDHI (R132) mutation or the glioma-CpG island methylator phenotype (G-CIMP) were obtained from the GEO data portal (http://www.ncbi.nlm.nih.gov/geo/) from the GSE16011 (n=30) (14) and GSE43388 $(n=15)(15)$ datasets. The $1 \mathrm{p} / 19 \mathrm{q}$ status was available for all samples. For each sample, CIC mRNA expression levels were determined using the $\log _{2}$-transformed RMA-normalized signal intensity from the Affymetrix Human Genome Plus 2.0 Microarray platform. In addition, expression profiles for 8 non-tumor brain tissues from the GSE16011 dataset were downloaded as controls.

Cross-dataset/platform mRNA data processing. To account for differences in systematic measurement between the distinct datasets and platforms, each set of CIC mRNA expression data was independently standardized by transforming the expression data to a mean of 0 and a standard deviation of 1 , as per a previously described method (16).

Gene set enrichment analysis (GSEA). To assess the enrichment of gene sets within different subgroups, GSEA 
was performed with 1,000 permutations, as previously described (17). Gene sets derived from the Biological Process Ontology (Molecular Signatures Database; http://software. broadinstitute.org/gsea/msigdb) were evaluated.

Statistical analysis. Overall survival (OS) time was defined as the time from diagnosis until mortality or the final follow-up. Survival data were analyzed using the Kaplan-Meier method and compared using the log-rank test. Univariate Cox regression analysis was performed to investigate the association between variables and OS time. A multiple Cox regression model was applied to investigate the independence of each prognostic indicator that was statistically significant within univariate Cox models. The associations between clinical and molecular variables were evaluated using a $\chi^{2}$ test or Fisher's exact test. Differences in CIC expression between groups were assessed by a Student's t-test or Mann-Whitney U-test. The optimal threshold to separate patients into the poor or good prognostic subgroups was determined by the maximally selected rank statistics (18). All calculations were performed with SPSS software (version 19.0; IBM Corporation, New York, NY, USA) and R software (version 3.2.5; https://www.r-project. org/). $\mathrm{P}<0.05$ was considered to indicate a statistically significant difference.

\section{Results}

Expression pattern of CIC among IDH-mutant oligodendroglial tumors. The demographical and molecular characteristics of all included patients are presented in Table I.

By comparing CIC mRNA expression between tumor and non-tumor brain tissue from TGCA, it was identified that CIC mutations were associated with decreased CIC mRNA expression levels (unpaired t-test, $\mathrm{P}<0.001$; data not shown), and were almost exclusively observed in tumors with $1 \mathrm{p} / 19 \mathrm{q}$ co-deletion (Fisher's exact test, $\mathrm{P}<0.001$; data not shown). Additionally, $C I C$ mRNA expression was increased in patients aged $<50$ years compared with those $\geq 50$, but no significant difference was observed between $C I C$ mRNA expression and sex (Fig. 1A).

In the GEO samples, $C I C$ expression was identified as significantly repressed in IDH-mutant oligodendroglial tumors relative to non-tumor tissue (unpaired t-test, $\mathrm{P}<0.05$ ). Among tumors with distinct clinical or molecular features, OAs were associated with increased $C I C$ mRNA expression compared with ODs, whereas no significant differences in $C I C$ expression between grade II and III tumors were identified. Regarding 1p/19q status, CIC mRNA expression was significantly decreased in tumors with $1 \mathrm{p} / 19 \mathrm{q}$ co-deletion compared with those without (Fig. 1B).

In the IHC examination of the tumor samples, it was observed that $16(30 \%), 13(24 \%), 18(33 \%)$ and $7(13 \%)$ cases were detected with absent, weak, moderate and strong nuclear expression of CIC, respectively (Fig. 2). All 4 cases with discrepancy were defined as weakly $(n=3)$ or moderately $(n=1)$ stained tumors after discussion (Three tumors were initially defined as moderately stained by one investigator but weakly stained by the other, while one tumor was initially defined as strongly stained by one investigator but moderately stained by the other). The protein expression patterns were consistent
Table I. Characteristics of patients with IDH-mutant oligodendroglial tumors.

\begin{tabular}{|c|c|c|c|}
\hline Variables & Xijing & TCGA & GEO \\
\hline Sample size, $\mathrm{n}$ & 54 & 265 & 45 \\
\hline \multicolumn{4}{|l|}{ World Health } \\
\hline \multicolumn{4}{|c|}{ Organization grade, $\mathrm{n}$} \\
\hline II & 22 & 163 & 6 \\
\hline III & 32 & 102 & 39 \\
\hline \multicolumn{4}{|l|}{ Tumor type, $\mathrm{n}$} \\
\hline OAs & 23 & 110 & 12 \\
\hline ODs & 31 & 155 & 33 \\
\hline \multicolumn{4}{|l|}{ Sex, $n$} \\
\hline Male & 29 & 143 & 29 \\
\hline Female & 25 & 122 & 16 \\
\hline \multicolumn{4}{|l|}{ Age, years } \\
\hline Median (range) & 43 & 41 & 45 \\
\hline & $(18-69)$ & $(18-74)$ & $(28-75)$ \\
\hline$<50, \mathrm{n}$ & 35 & 184 & 27 \\
\hline$\geq 50, \mathrm{n}$ & 19 & 81 & 18 \\
\hline \multicolumn{4}{|c|}{$\begin{array}{l}\text { Karnofsky } \\
\text { performance status }\end{array}$} \\
\hline Median (range) & $\begin{array}{c}80 \\
(50-100)\end{array}$ & $\begin{array}{c}90 \\
(60-100)\end{array}$ & $\begin{array}{c}100 \\
(10-100)\end{array}$ \\
\hline$<80, \mathrm{n}$ & 21 & 3 & 8 \\
\hline$\geq 80, \mathrm{n}$ & 28 & 59 & 26 \\
\hline \multicolumn{4}{|c|}{ IDH mutation type, $\mathrm{n}$} \\
\hline$I D H I$ & 54 & 247 & $45^{\mathrm{a}}$ \\
\hline$I D H 2$ & 0 & 18 & - \\
\hline \multicolumn{4}{|l|}{ 1p/19q status, $n$} \\
\hline Co-deletion & 28 & 153 & 29 \\
\hline Intact & 26 & 112 & 16 \\
\hline \multicolumn{4}{|c|}{$C I C$ mutation status, $\mathrm{n}$} \\
\hline CIC mutant & - & 104 & - \\
\hline$C I C$ wide-type & - & 161 & - \\
\hline
\end{tabular}

${ }^{\text {aTh }}$ IDH1 mutation status of three samples was predicted by glioma CpG island methylator phenotype. '-' indicates that the variables were unknown or untested. OAs, oligoastrocytomas; ODs, oligodendrogliomas; IDH, isocitrate dehydrogenase; CIC, capicua transcriptional repressor; TCGA, The Cancer Genome Atlas; GEO, Gene Expression Omnibus.

with the mRNA levels from the GEO and TGCA data (Fig. 1). Notably, absent CIC expression was significantly associated with $1 \mathrm{p} / 19 \mathrm{q}$ co-deleted tumors $\left(\chi^{2}\right.$ test, $\left.\mathrm{P}<0.001\right)$ and ODs $\left(\chi^{2}\right.$ test, $\mathrm{P}=0.006)$, whereas strong expression was associated with tumors without $1 \mathrm{p} / 19 \mathrm{q}$ co-deletion ( $\chi^{2}$ test, $\mathrm{P}=0.047$; Fig. $1 \mathrm{C}$ ).

Increased CIC expression is associated with a favorable prognosis in IDH-mutant, 1p/19q co-deletedoligodendroglial tumors. Of the 153 oligodendroglial tumors from TCGA with an $I D H$ mutation and $1 \mathrm{p} / 19 \mathrm{q}$ co-deletion, $C I C$ mutations were detected in $100(65.4 \%)$ cases. No significant association was 

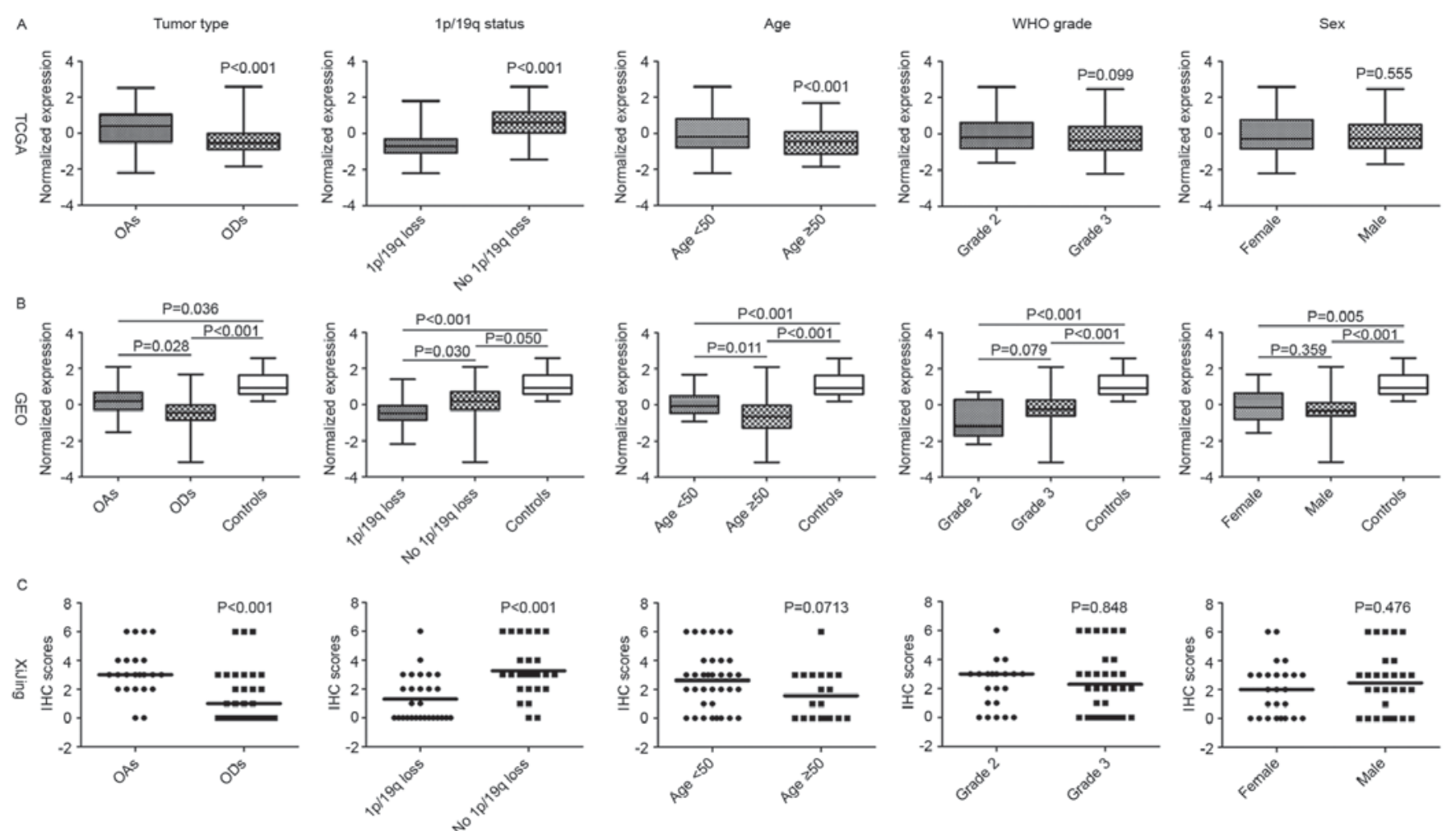

Figure 1. The mRNA and protein expression levels of CIC within each subgroup. A Student's t-test was used to compare the mRNA expression levels of samples from (A) TCGA and (B) GEO, and a Mann-Whitney U-test was used to compare the IHC scores from (C) the Xijing cohort. CIC, capicua transcriptional repressor; TCGA, The Cancer Genome Atlas; GEO, Gene Expression Omnibus; IHC, immunohistochemistry; OAs, oligoastrocytomas; ODs, oligodendrogliomas; WHO, World Health Organization.

A

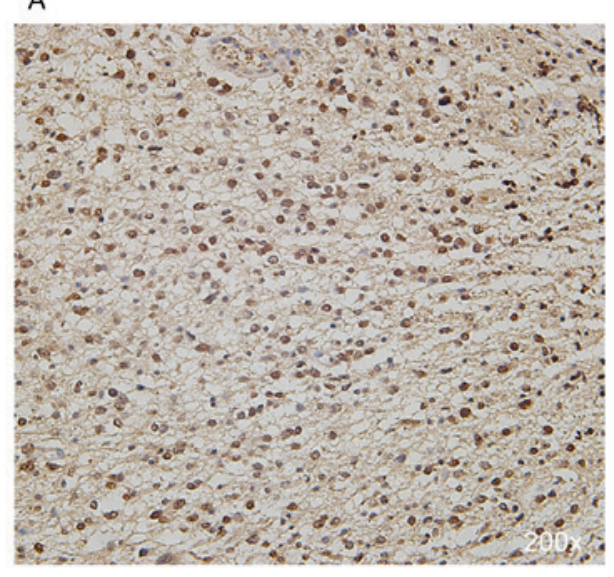

B

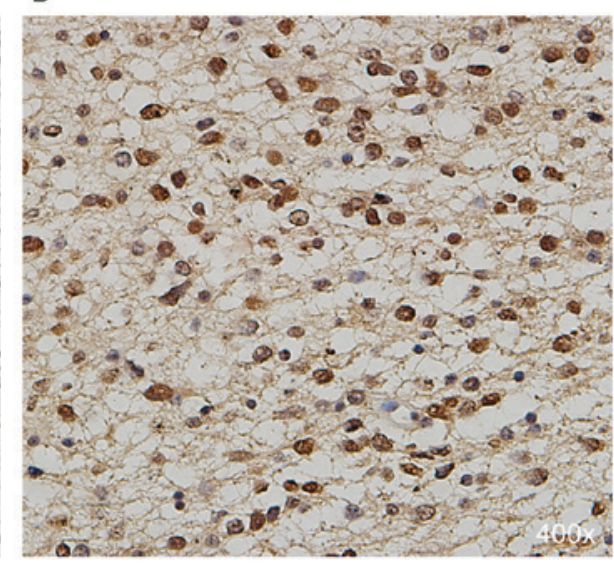

Figure 2. Representative images of immunohistochemical staining for CIC in oligodendroglial tumors. Positive CIC expression with strong nuclear staining under (A) x200 and (B) x400 magnification. CIC, capicua transcriptional repressor.

identified between $C I C$ mutations and OS time among TCGA samples (log-rank $\mathrm{P}=0.225$ ). However, increased CIC expression was associated with a favorable prognosis in $I D H$-mutant oligodendroglial tumors with $1 \mathrm{p} / 19 \mathrm{q}$ co-deletion (median OS time, higher vs. lower, not reached vs. 136.1 months; log-rank $\mathrm{P}=0.034$; Fig. 3A). Similarly, among $I D H$-mutant and $1 \mathrm{p} / 19 \mathrm{q}$ co-deleted oligodendroglial tumors from GEO, higher CIC expression was associated with longer OS time compared with lower CIC expression (median OS time, higher vs. lower, 119.5 vs. 45.7 months; log-rank $\mathrm{P}=0.012$; Fig. 3B). Additionally, in the IHC examination of the present study cohort, patients with absent CIC expression exhibited a significantly shorter OS than those with detectable protein expression (weak, moderate or strong; median OS, absent vs. detectable, 75.0 vs. 42.2 months; log-rank $\mathrm{P}=0.029$; Fig. 3C). These data collectively demonstrate that higher CIC expression is a favorable indicator for OS time among patients with oligodendroglial tumors with $I D H$ mutation and $1 \mathrm{p} / 19 \mathrm{q}$ co-deletion.

Higher CIC expression is associated with poor prognosis in IDH-mutant oligodendroglial tumors without the $1 p / 19 q$ 

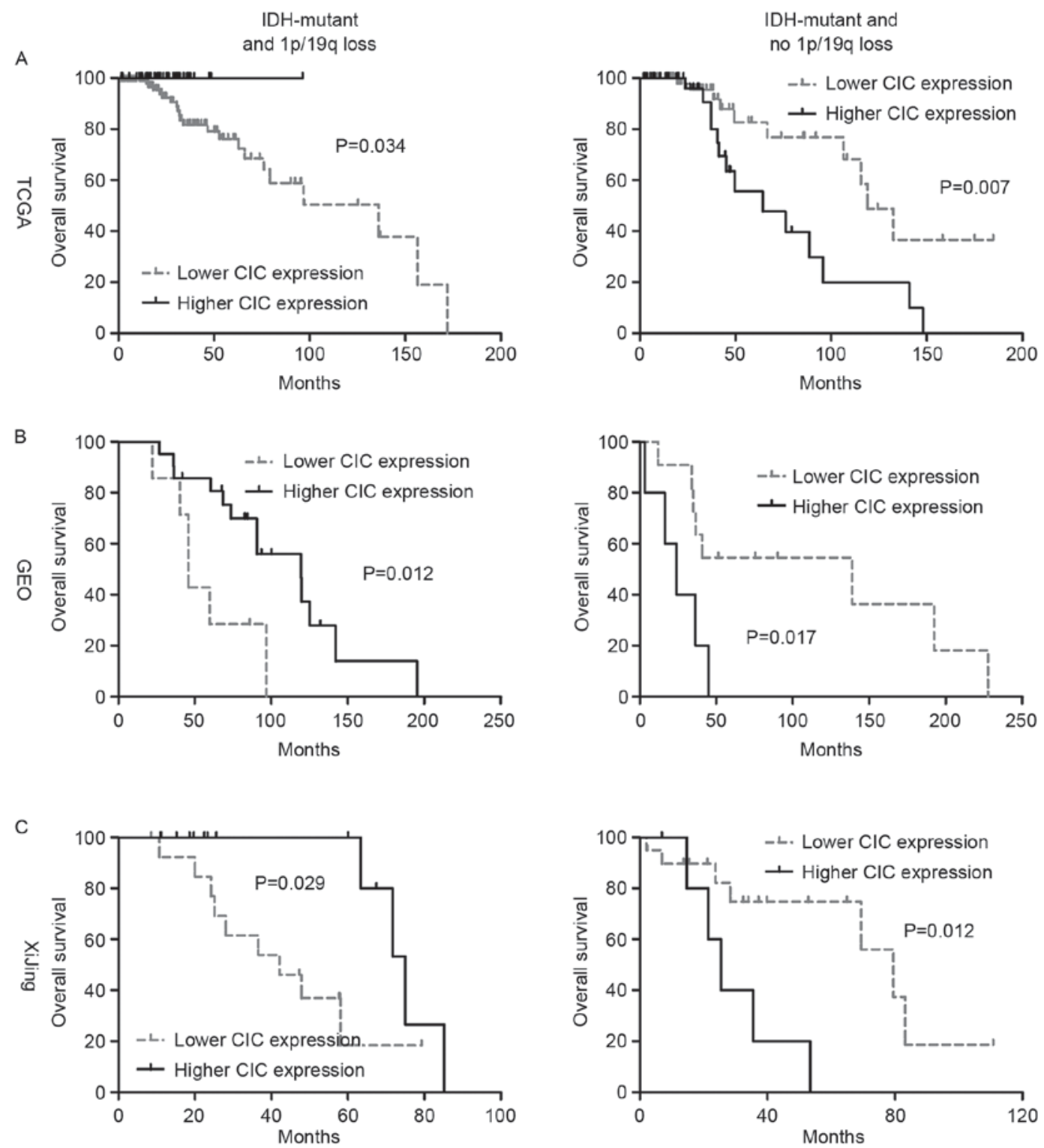

Figure 3. Overall survival difference between subgroups with different CIC mRNA or protein expression levels. Overall survival analysis of patients in the (A) TCGA, (B) GEO and (C) Xijing cohorts. CIC, capicua transcriptional repressor; TCGA, The Cancer Genome Atlas; GEO, Gene Expression Omnibus; IDH, isocitrate dehydrogenase.

co-deletion. In contrast to IDH-mutant, $1 \mathrm{p} / 19 \mathrm{q}$ co-deleted oligodendroglial tumors, only 4 cases $(3.6 \%)$ were detected with CIC mutations in those without 1p/19q co-deletion from TCGA. In this subset of oligodendroglial tumors, higher CIC expression was associated with poorer OS (median OS, higher vs. lower, 64.4 vs. 119.0 months; log-rank $\mathrm{P}=0.007$; Fig. 3A). The distinct prognostic ability was also confirmed in GEO samples (median OS, higher vs. lower, 23.8 vs. 138.8 months; log-rank $\mathrm{P}=0.017$; Fig. 3B). Additionally, at the protein level, high nuclear CIC expression was also significantly associated with poorer OS (median OS, strong vs. lower or absent, 25.4 vs. 79.6 months; log-rank P=0.012; Fig. 3C). Therefore, the data collectively indicated that higher CIC expression is an unfavorable indicator for OS time among patients with oligodendroglial tumors with $I D H$ mutations and no $1 \mathrm{p} / 19 \mathrm{q}$ co-deletion.

Bioinformatics analysis. To explore the biological mechanisms for the observed distinct prognostic role of CIC expression level, the expression levels of previously identified genes targeted by CIC [ETS variant (ETV)1, ETV4, ETV5 and Cyclin D1] were compared within TCGA samples. It was demonstrated that among $I D H$-mutant, $1 \mathrm{p} / 19 \mathrm{q}$ co-deleted oligodendroglial tumors, all the targets were significantly upregulated in the lower expression group compared with the higher expression groups (Fig. 4A), suggesting a decrease in the transcriptional repressor activity of CIC; whereas among the IDH-mutant, $1 \mathrm{p} / 19 \mathrm{q}$ intact tumors, the expression of the target genes was not significantly altered (Fig. 4B), suggesting that the role of $C I C$ in this subset of gliomas may be independent of its transcriptional regulatory activity. Furthermore, GSEA revealed that among the IDH-mutant, $1 \mathrm{p} / 19 \mathrm{q}$ co-deleted tumors, a reduction in $C I C$ expression was associated with the enrichment of gene signatures involved in the biosynthesis of RNA and protein, including translational initiation $(\mathrm{P}<0.0001)$ and biosynthetic process $(\mathrm{P}<0.0001)$. By contrast, among those tumors without $1 \mathrm{p} / 19 \mathrm{q}$ co-deletion, the increase in CIC expression was 


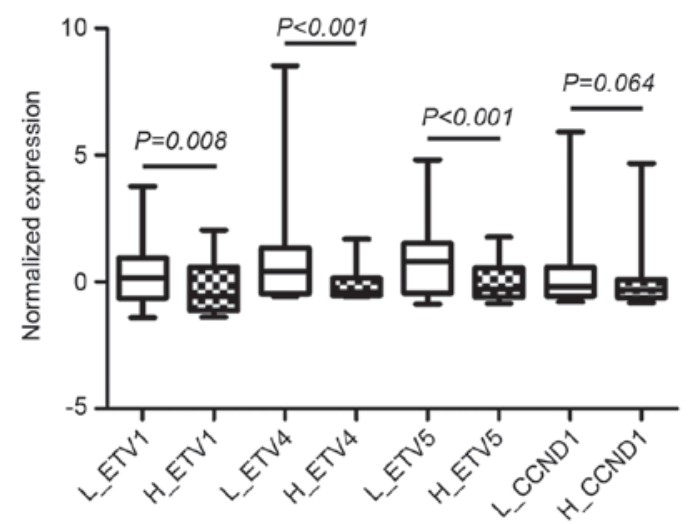

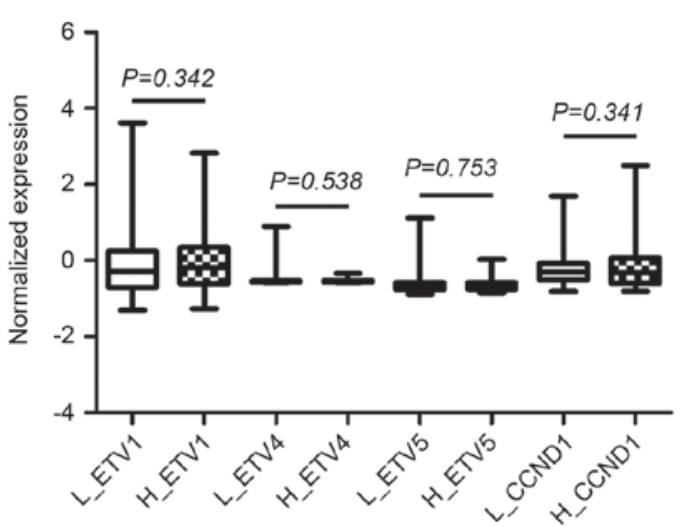

Figure 4. The mRNA expression levels of CIC target genes (ETV1, ETV4, ETV5 and CCND1) within low and high CIC expression subgroups among (A) IDH-mutant, 1p/19q co-deleted oligodendroglial tumors and (B) IDH-mutant, 1p/19q not co-deleted oligodendroglial tumors in The Cancer Genome Atlas dataset. CIC, capicua transcriptional repressor; ETV, ETS variant; CCND1, Cyclin D1; L_, low CIC expression group; H_, high CIC expression group; IDH, isocitrate dehydrogenase.

associated with the enrichment of gene signatures involved in cell proliferation, including regulation of mitosis (Normalized enrichment score $[\mathrm{NES}]=-1.74, \mathrm{P}=0.041)$, treatment resistance, including nucleotide excision repair $(\mathrm{NES}=-1.52, \mathrm{P}=0.050)$ and epigenetic modification, including chromatin modification (NES=-1.77, $\mathrm{P}=0.016)$.

Alerted CIC expression is an independent predictor for OS time among IDH-mutant, 1p/19q-intact oligodendroglial tumors, but not among $1 p / 19 q$ co-deleted tumors. Within $I D H$-mutant and 1p/19q co-deleted oligodendroglial tumors, a univariate Cox regression model revealed that patient age and CIC expression groups (for the GEO and IHC cohorts) were significantly associated with OS, whereas WHO grade, histological type, Karnofsky performance status and CIC mutations were not (Table II). The largely consistent results from all cohorts supported the robustness of this finding. However, the multivariate Cox model demonstrated that only patient age was consistently an independent indicator (Table II).

The multivariate Cox model was not applied to $I D H$-mutant, 1p/19q-intact oligodendroglial tumors as only CIC expression group consistently reached statistical significance in each cohort in the univariate Cox regression models (Table III). The data suggested that $C I C$ expression groups may be an independent prognostic factor in this subset of gliomas; however, further validation is required.

Development of a simple classification scheme based on CIC expression level for IDH-mutant oligodendroglial tumors. The combination of $1 \mathrm{p} / 19 \mathrm{q}$ status and CIC expression stratified all patients with $I D H$-mutant oligodendroglial tumors into 4 prognostic subgroups: Subgroup A (lower CIC expression in $1 \mathrm{p} / 19 \mathrm{q}$ co-deleted tumors), subgroup $B$ (higher CIC expression in $1 \mathrm{p} / 19 \mathrm{q}$ co-deleted tumors), subgroup $C$ (lower CIC expression in 1p/19q-intact tumors) and subgroup $D$ (higher CIC expression in $1 \mathrm{p} / 19 \mathrm{q}$-intact tumors). Survival analysis revealed that patients within subgroups $B$ and $C$ exhibited the longest overall survival times, and patients in subgroups $D$ the shortest, followed by subgroup $A$ (Fig. 5).
Accordingly, by comparing $C I C$ expression levels within the subgroups, subgroups $A$ and $D$ demonstrated the lowest and highest $C I C$ expression, respectively, whereas subgroups $B$ and $C$ were observed to possess similar, intermediate expression levels (Fig. 5). Therefore, a simple classification scheme was devised based on the CIC expression of IDH-mutant oligodendroglial tumors, with the thresholds of the upper expression boundary of subgroup $A$ and the lower expression boundary of subgroup $D$. According to this novel classification scheme, all patients were classified into 3 distinct prognostic subgroups regardless of the $1 \mathrm{p} / 19 \mathrm{q}$ status; patients with intermediate CIC expression exhibited the longest survival times, followed by those with low expression, whereas patients with high CIC expression had the shortest survival times (Fig. 5). In summary, this novel prognostic scheme may be useful for optimizing the risk classification of $I D H$-mutant oligodendroglial tumors.

\section{Discussion}

The CIC gene has long been implicated in the pathogenesis of oligodendroglial tumors (10). Recurrent mutations in this gene were frequently observed in a subset of gliomas with $I D H$ mutations and $1 \mathrm{p} / 19 \mathrm{q}$ co-deletion. Studies have demonstrated the critical role of the genetic alterations to this gene in determining tumor biological and clinical behaviors $(3,4)$. However, no consistent results have been reported regarding the association between the genetic alterations and prognosis, which critically compromises its clinical utility $(7,9)$. The results of the present study revealed that, despite the overall reduced expression level, $C I C$ mRNA expression was variable across $I D H$-mutant oligodendroglial tumors. It was observed that the $C I C$ expression level was associated with different tumor types (OA or OD), the $1 \mathrm{p} / 19 \mathrm{q}$ status and patient ages. The variable expression level also reflected the genetic status of $C I C$; due to the frequent loss of one copy of chromosome arm $19 q$, subsequent inactivating mutations in gene coding regions of the remaining copy may result in gene repression (2). Therefore, the CIC expression pattern may be useful as an 


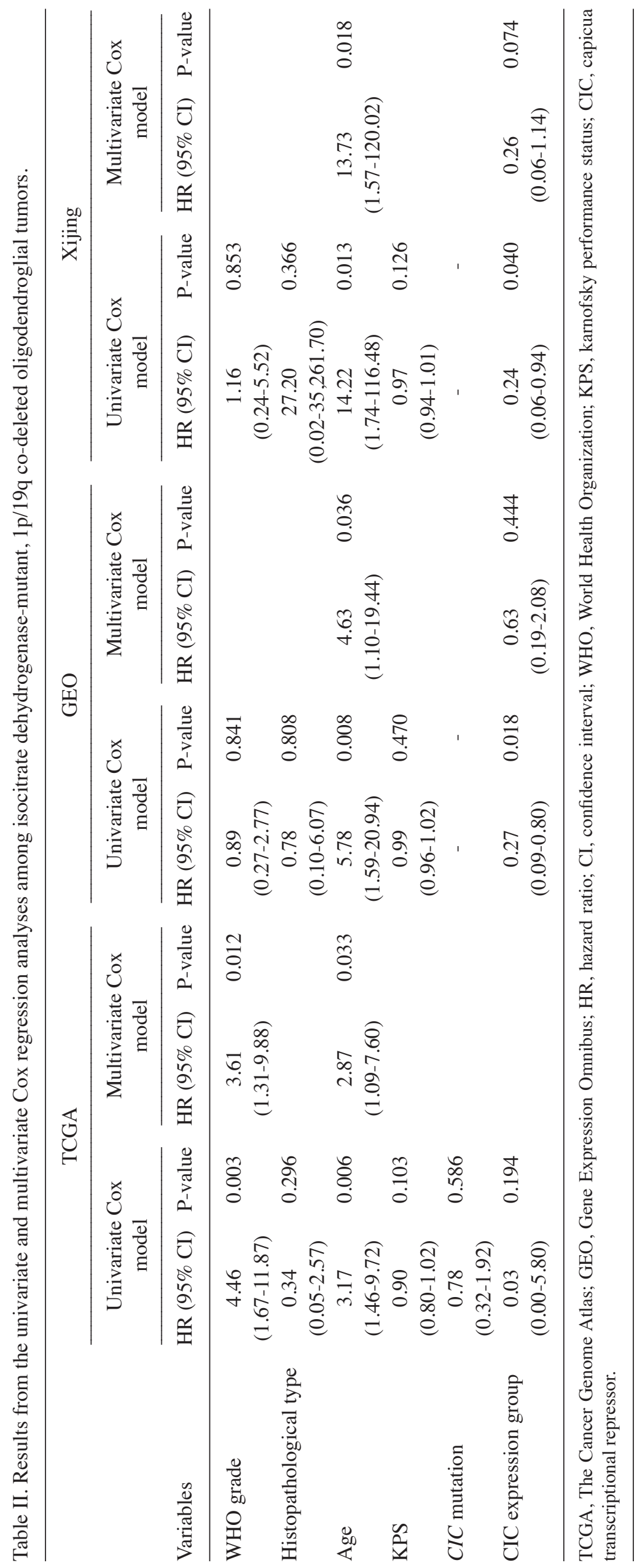


Table III. Results from the univariate Cox regression analysis among isocitrate dehydrogenase-mutant, 1p/19q intact oligodendroglial tumors.

\begin{tabular}{|c|c|c|c|c|c|c|}
\hline \multirow[b]{2}{*}{ Variables } & \multicolumn{2}{|c|}{ TCGA } & \multicolumn{2}{|l|}{ GEO } & \multicolumn{2}{|c|}{ Xijing } \\
\hline & HR $(95 \%$ CI $)$ & P-value & HR $(95 \% \mathrm{CI})$ & P-value & HR $(95 \% \mathrm{CI})$ & P-value \\
\hline WHO grade & $1.00(0.39-2.54)$ & 0.994 & $0.92(0.12-7.35)$ & 0.940 & $2.81(0.75-10.49)$ & 0.124 \\
\hline Histopathologic type & $0.97(0.43-2.21)$ & 0.950 & $1.31(0.41-4.18)$ & 0.644 & $0.54(0.15-2.02)$ & 0.360 \\
\hline Age & $0.91(0.27-3.90)$ & 0.875 & $1.99(0.51-7.76)$ & 0.323 & $0.82(0.10-6.73)$ & 0.857 \\
\hline KPS & $0.66(0.13-3.29)$ & 0.610 & $0.97(0.93-1.01)$ & 0.092 & $0.99(0.94-1.04)$ & 0.662 \\
\hline CIC mutation & $0.45(0.10-2.00)$ & 0.292 & - & - & - & - \\
\hline CIC expression group & $2.94(1.29-6.67)$ & 0.010 & $4.15(1.17-14.73)$ & 0.028 & $4.66(1.24-17.48)$ & 0.023 \\
\hline
\end{tabular}

'-' indicates that the variables were unknown or untested. TCGA, The Cancer Genome Atlas; GEO, Gene Expression Omnibus; HR, hazard ratio; CI, confidence interval; WHO, World Health Organization; KPS, karnofsky performance status; CIC, capicua transcriptional repressor.
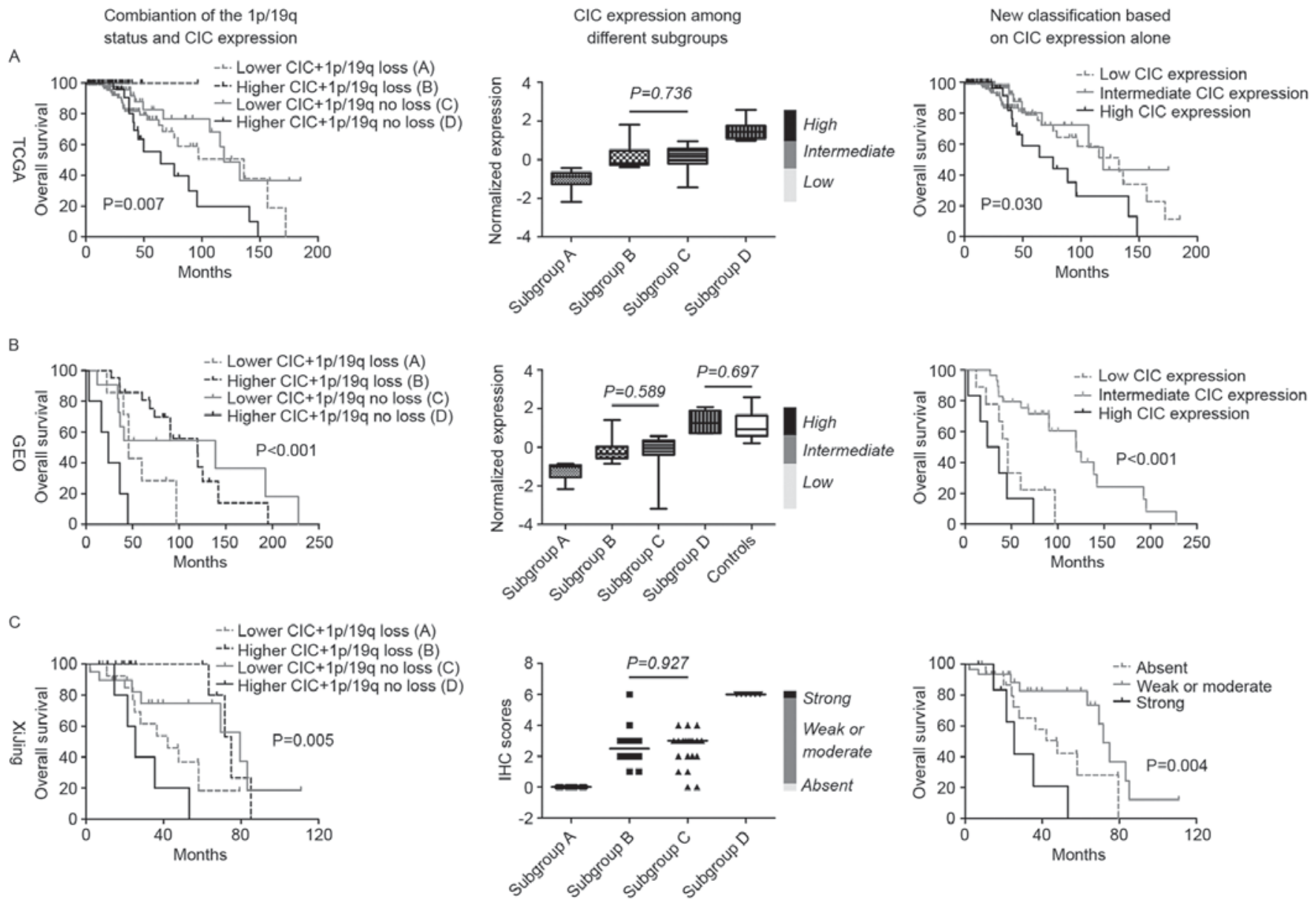

Figure 5. Novel classification schemes for optimizing the accuracy of prognosis for patients with isocitrate dehydrogenase-mutant oligodendroglial tumors. The co-assessment of the 1p/19q status and CIC (mRNA and protein) expression levels was used to stratify patients into 4 distinct prognostic subgroups (subgroups $A-D$ ) in the (A) TGCA, (B) GEO and (C) Xijing cohorts. By comparing the expression levels of CIC within the subgroups, a novel classification model was created based on CIC expression levels in which patients are classified into 3 distinct prognostic subgroups using the upper expression boundary of subgroup $A$ and the lower expression boundary of subgroup $D$ as thresholds. CIC, capicua transcriptional repressor; TCGA, The Cancer Genome Atlas; GEO, Gene Expression Omnibus.

alternative to detecting somatic mutations in predicting the prognosis of patients with oligodendroglial tumors.

To the best of our knowledge, the present study demonstrated for the first time the distinct prognostic value of altered $C I C$ expression depending on $1 \mathrm{p} / 19 \mathrm{q}$ status among $I D H$-mutant oliogendroglial tumors. Among tumors carrying the $1 \mathrm{p} / 19 \mathrm{q}$ co-deletion, higher CIC expression (at mRNA and protein levels) was a favorable indicator of survival, 
suggesting a tumor-suppressive role in that subset of gliomas. The decreased expression of CIC predominantly results from inactivating mutations in gene coding regions and the rapid degradation of mutant proteins (3). Decreased CIC expression is associated with a decrease in CIC transcriptional repressor activity and thus may have contributed to tumor aggression via the induction of a transcriptional profile of high activity in transcription and translation, in line with the GSEA results of the present study. Recently, Gleize et al (4) demonstrated similar results regarding the tumor-suppressive role of wild-type $C I C$, but with a focus on its mutation status in $I D H$-mutant, 1p/19q co-deleted oligodendroglial tumors. Mutant proteins were quickly adequately degraded in those tumors, resulting in increased CIC expression in certain cases (4). A previous study identified that mutant proteins decrease cell clonogenicity and slow tumor growth in the presence of mutant $I D H$ proteins via the regulation of cytosolic citrate metabolism (19). In such cases, tumors with the increased expression of mutant CIC may be associated with favorable OS. These observations support the hypothesis that quantifying CIC expression may be more useful than determining its mutation status for providing accurate prognostic information in this subset of gliomas.

Until recently, only a small number of studies focused on the roles of $C I C$ alterations, including somatic mutations and abnormal expression, in IDH-mutant, 1p/19q-intact oligodendroglial tumors. In line with previous studies $(3,8,9)$, the results of the present study revealed a low incidence of $C I C$ mutations and no significant association between CIC mutation and overall survival time in this subset of tumors, from TCGA. In the present study, higher CIC expression (at mRNA and protein levels) was associated with poor prognosis in IDH-mutant, 1p/19q-intact oligodendroglial tumors. This suggested a pro-oncogenic role for $C I C$, particularly the wild type, in the molecular context of $I D H$ mutations and 1p/19-intact. Additionally, in this subset of gliomas, the tumor-promoting role of CIC was possibly independent of its transcriptional repression activity, as evidenced by the similar expression levels of its targets among tumors with different levels of $C I C$ expression. GSEA analysis revealed that, in the presence of intact $1 \mathrm{p} / 19 \mathrm{q}$, increased $C I C$ expression was associated with the activation of proliferative pathways. Limited data is available concerning the oncogenic role of wild-type CIC in cancer biology. CIC was rarely observed to be mutated although it was highly expressed in medulloblastoma, suggesting its relevance in tumor genesis and progression $(3,20)$. Furthermore, a previous study demonstrated that wild-type CIC protein may rescue the reduction in cell proliferation caused by $I D H I$ mutations in the presence of intact $1 \mathrm{p} / 19 \mathrm{q}$ (19). The aforementioned data, along with the data of the present study, suggest a potential oncogenic role for wild-type $C I C$ in human cancer; however, further investigation is required.

The equivocal histopathologic definition of oligodendroglial tumors results in inefficiency in their management (2). Molecular markers have demonstrated promising value for optimizing the prediction of prognosis for patients with these types of glioma (1). In the present study, the distinct prognostic values of $C I C$ expression with regard to the $1 p / 19 q$ status among IDH-mutant oligodendroglial tumors was demonstrated. The combination of altered CIC expression and the
$1 \mathrm{p} / 19 \mathrm{q}$ status yielded 4 distinct prognostic subgroups and provided the optimized prediction of prognosis for patients with $I D H$-mutant oligodendroglial tumors. Furthermore, by comparing the expression levels of $C I C$ within the subgroups, it was identified that regardless of the $1 p / 19 q$ status, CIC expression was associated with distinct prognoses; tumors with intermediate CIC expression were associated with the best outcomes, whereas those with the lowest CIC expression had less favorable outcomes and those with the highest expression had the poorest. In addition, the results from the IHC cohort suggested that this novel scheme may be accomplished by a simple but widely-used test method in the clinical setting. Therefore, a novel classification scheme based only on CIC expression level may exhibit clinical utility by providing a simple way to rapidly estimate the prognosis of patients with IDH-mutant oligodendroglial tumors without the assessment of $1 \mathrm{p} / 19 \mathrm{q}$ status.

The reproducibility and consistency of the distinct prognostic value of $C I C$ expression at the mRNA and protein levels across the cohorts suggest that the results of the present study are unlikely to be spurious due to technical artifacts or sample bias. However, the significance of the present study has a number of limitations. Neither mRNA quantification nor the IHC method were able to distinguish between the mutant and wide-type $C I C$, which brought uncertainty into data interpretation. In addition, the sample size of the IHC cohort was limited, and cytoplasmic CIC staining was not considered in IHC scoring.

In conclusion, to the best of our knowledge, the present study demonstrated for the first time the distinct prognostic value of $C I C$ expression with regard to the $1 \mathrm{p} / 19 \mathrm{q}$ status among $I D H$-mutant oligodendroglial tumors. The novel classification scheme based on the CIC expression level may be clinically useful by providing a simple and rapid estimation for the prognosis of patients with IDH-mutant oligodendroglial tumors. This study also highlighted the necessity of further investigation into the heterogeneous role of CIC, either in mutant or wild-type forms, in oligodendroglial tumors.

\section{Acknowledgements}

The authors wish to thank Mrs. Jun-li Huo, and Mrs. Juan Li (Department of Neurosurgery, Xijing Hospital) for collecting and registering the samples and Dr Bo-lin Liu (Division of Neurosurgery, Arrowhead Regional Medical Center, Colton, CA, USA) for revising the manuscript. The present study was partially funded by the Natural Science Basic Research Plan in Shaanxi Province of China (grant no. 2014JM4099).

\section{References}

1. Cancer Genome Atlas Research Network, Brat DJ, Verhaak RG, Aldape KD, Yung WK, Salama SR, Cooper LA, Rheinbay E, Miller CR, Vitucci M, et al: Comprehensive, integrative genomic analysis of diffuse lower-grade gliomas. N Engl J Med 372: 2481-2498, 2015

2. Wesseling P, van den Bent M and Perry A: Oligodendroglioma: Pathology, molecular mechanisms and markers. Acta Neuropathol 129: 809-827, 2015.

3. Sahm F, Koelsche C, Meyer J, Pusch S, Lindenberg K, Mueller W, Herold-Mende C, von Deimling A and Hartmann C: CIC and FUBP1 mutations in oligodendrogliomas, oligoastrocytomas and astrocytomas. Acta Neuropathol 123: 853-860, 2012. 
4. Gleize V, Alentorn A, Connen de Kérillis L, Labussière M, Nadaradjane AA, Mundwiller E, Ottolenghi C, Mangesius S, Rahimian A, Ducray F, et al: CIC inactivating mutations identify aggressive subset of 1p19q codeleted gliomas. Ann Neurol 78: $355-374,2015$.

5. van den Bent MJ, Brandes AA, Taphoorn MJ, Kros JM, Kouwenhoven MC, Delattre JY, Bernsen HJ, Frenay M, Tijssen CC, Grisold W, et al: Adjuvant procarbazine, lomustine and vincristine chemotherapy in newly diagnosed anaplastic oligodendroglioma: Long-term follow-up of EORTC brain tumor group study 26951. J Clin Oncol 31: 344-350, 2013.

6. Reuss DE, Sahm F, Schrimpf D, Wiestler B, Capper D, Koelsche C, Schweizer L, Korshunov A, Jones DT, Hovestadt V, et al: ATRX and IDH1-R132H immunohistochemistry with subsequent copy number analysis and IDH sequencing as a basis for an 'integrated' diagnostic approach for adult astrocytoma, oligodendroglioma and glioblastoma. Acta Neuropathol 129: 133-146, 2015.

7. Jiao Y, Killela PJ, Reitman ZJ, Rasheed AB, Heaphy CM, de Wilde RF, Rodriguez FJ, Rosemberg S, Oba-Shinjo SM Nagahashi Marie SK, et al: Frequent ATRX, CIC, FUBP1 and IDH1 mutations refine the classification of malignant gliomas. Oncotarget 3: 709-722, 2012.

8. Yip S, Butterfield YS, Morozova O, Chittaranjan S, Blough MD, An J, Birol I, Chesnelong C, Chiu R, Chuah E, et al: Concurrent CIC mutations, IDH mutations and $1 \mathrm{p} / 19 \mathrm{q}$ loss distinguish oligodendrogliomas from other cancers. J Pathol 226: 7-16, 2012.

9. Chan AK, Pang JC, Chung NY, Li KK, Poon WS, Chan DT, Shi Z, Chen L, Zhou L and Ng HK: Loss of CIC and FUBP1 expressions are potential markers of shorter time to recurrence in oligodendroglial tumors. Mod Pathol 27: 332-342, 2014.

10. Bettegowda C, Agrawal N, Jiao Y, Sausen M, Wood LD, Hruban RH, Rodriguez FJ, Cahill DP, McLendon R, Riggins G, et al: Mutations in CIC and FUBP1 contribute to human oligodendroglioma. Science 333: 1453-1455, 2011.

11. Jimenez G, Shvartsman SY and Paroush Z: The Capicua repressor-a general sensor of RTK signaling in development and disease. J Cell Sci 125: 1383-1391, 2012.

12. Fuller GN and Scheithauer BW: The 2007 revised World Health Organization (WHO) classification of tumours of the central nervous system: Newly codified entities. Brain Pathol 17: 304-307, 2007.
13. Chen X, Yun J, Fei F, Yi J, Tian R, Li S and Gan X: Prognostic value of nuclear hepatoma-derived growth factor (HDGF) localization in patients with breast cancer. Pathol Res Pract 208: 437-443, 2012.

14. Gravendeel LA, Kouwenhoven MC, Gevaert O, de Rooi JJ, Stubbs AP, Duijm JE, Daemen A, Bleeker FE, Bralten LB, Kloosterhof NK, et al: Intrinsic gene expression profiles of gliomas are a better predictor of survival than histology. Cancer Res 69: 9065-9072, 2009.

15. Erdem-Eraslan L, Gravendeel LA, de Rooi J, Eilers PH, Idbaih A, Spliet WG, den Dunnen WF, Teepen JL, Wesseling P, Sillevis Smitt PA, et al: Intrinsic molecular subtypes of glioma are prognostic and predict benefit from adjuvant procarbazine, lomustine and vincristine chemotherapy in combination with other prognostic factors in anaplastic oligodendroglial brain tumors: A report from EORTC study 26951. J Clin Oncol 31: 328-336, 2013.

16. Woo HG, Park ES, Cheon JH, Kim JH, Lee JS, Park BJ, Kim W, Park SC, Chung YJ, Kim BG, et al: Gene expression-based recurrence prediction of hepatitis B virus-related human hepatocellular carcinoma. Clin Cancer Res 14: 2056-2064, 2008.

17. Subramanian A, Tamayo P, Mootha VK, Mukherjee S, Ebert BL Gillette MA, Paulovich A, Pomeroy SL, Golub TR, Lander ES and Mesirov JP: Gene set enrichment analysis: A knowledge-based approach for interpreting genome-wide expression profiles. Proc Natl Acad Sci USA 102: 15545-15550, 2005.

18. Hothorn T and Zeileis A: Generalized maximally selected statistics. Biometrics 64: 1263-1269, 2008.

19. Chittaranjan S, Chan S, Yang C, Yang KC, Chen V, Moradian A, Firme M, Song J, Go NE, Blough MD, et al: Mutations in CIC and IDH1 cooperatively regulate 2 -hydroxyglutarate levels and cell clonogenicity. Oncotarget, 2014

20. Lee CJ, Chan WI and Scotting PJ: CIC, a gene involved in cerebellar development and ErbB signaling, is significantly expressed in medulloblastomas. J Neurooncol 73: 101-108, 2005.

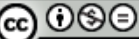

This work is licensed under a Creative Commons Attribution-NonCommercial-NoDerivatives 4.0 International (CC BY-NC-ND 4.0) License. 